\title{
A rare etiology of idiopathic recurrent acute pancreatitis
}
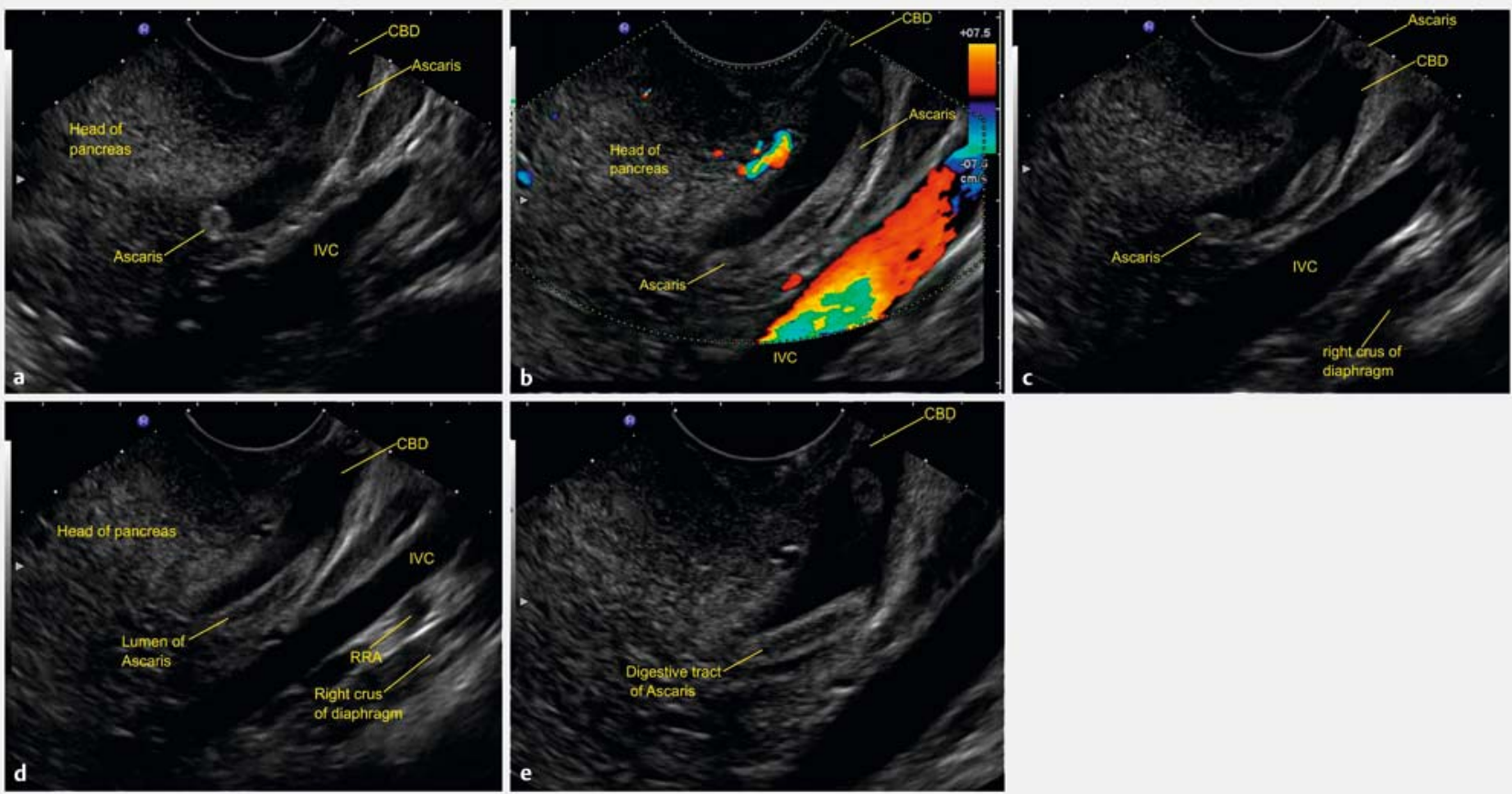

Fig. 1 Linear endoscopic ultrasound (EUS) images. a EUS from the duodenal bulb showed a round echogenic shadow in the common bile duct (CBD). b A linear echogenic structure without acoustic shadow was seen in the CBD. On color Doppler, blood flow could be seen in the inferior vena cava (IVC). $\mathbf{c}$ The linear echogenic shadow was seen coiling in the CBD. d, e The linear structure had two hyperechoic linear strips on either side of the longitudinal anechoic lumen, representing the fluid-filled digestive tract of the worm ("double tube sign" or "inner tube sign"). RRA, right renal artery.

A 30-year-old Indian woman was referred with recurrent episodes of acute pancreatitis; she had experienced four episodes in the previous 7 months. There was no history of alcohol or drug intake. She was diagnosed with idiopathic recurrent acute pancreatitis (IRAP), as results of blood investigations, ultrasonography, computed tomography (abdomen), and magnetic resonance cholangiopancreatography were normal. Linear endoscopic ultrasound (EUS) was performed to diagnose the etiology of IRAP.

Linear EUS from the duodenal bulb showed a round echogenic shadow in the head of the pancreas ( $\boldsymbol{\nabla}$ Fig. $\mathbf{1} \mathbf{a}$ ). It also revealed a linear echogenic mobile structure without acoustic shadow in the common bile duct (CBD) ( $\triangleright$ Fig.1 $\mathbf{b}$ ). The linear echogenic mobile shadow was coil-

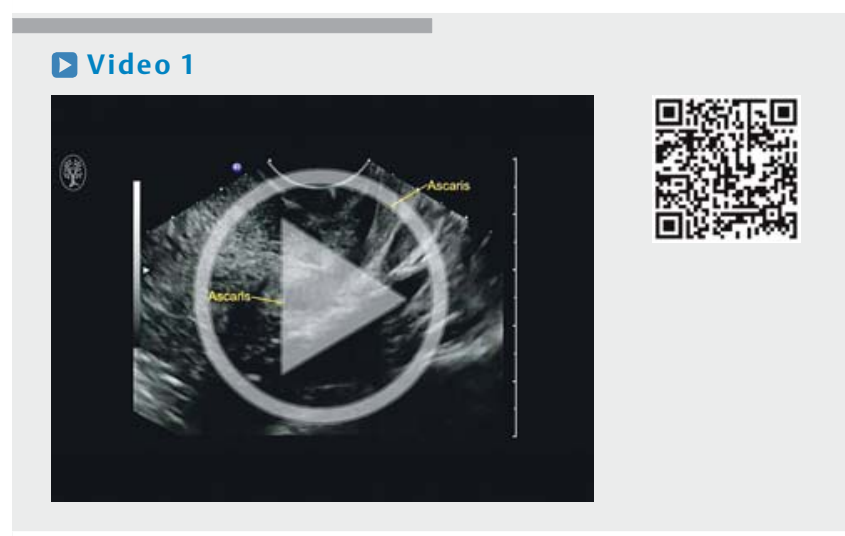

- Video 1: Linear endoscopic ultrasound from the duodenal bulb showed a linear echogenic mobile structure without acoustic shadow coiling in the common bile duct, with two hyperechoic linear strips on either side of the longitudinal anechoic lumen. On sideviewing endoscopy, one creamy-white worm was visualized outside the papilla; it was removed using biopsy forceps and identified as Ascaris lumbricoides. 


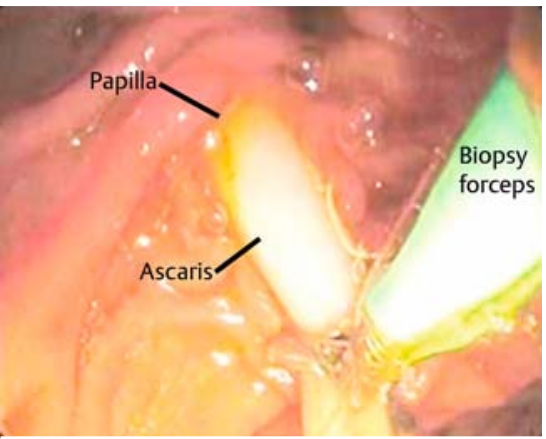

Fig. 2 (Still Image) On side-viewing endoscopy, one creamy-white worm was seen partially lying outside the papilla.

ing within the CBD, confirming the diagnosis of a biliary ascariasis ( $\triangleright$ Fig. 1 c). This linear structure had two hyperechoic linear strips on either side of the longitudinal anechoic lumen, representing the fluid-filled alimentary canal of the worm ("double tube sign" or "inner tube sign")

( $\triangleright$ Fig. 1d,e; $>$ Video 1). On side-viewing endoscopy, one creamy-white worm was seen, partially lying outside the papilla. The worm was extracted using biopsy forceps and identified as Ascaris lumbricoides ( $>$ Fig. 2 ; $>$ Video 1 ). The worm was $10 \mathrm{~cm}$ long. Subsequently, the patient received de-worming treatment with albendazole. At the follow-up visit, the patient was asymptomatic, and EUS revealed a normal CBD.
Ascaris lumbricoides infestation is endemic in tropical countries. Although most infections by roundworms are asymptomatic, they can produce diverse manifestations, including hepatobiliary and pancreatic complications [1]. Ascaris is a common etiology of acute pancreatitis in developing countries. The mechanism of ascariasis-induced acute pancreatitis includes obstruction of the ampulla of Vater, and invasion of the CBD or pancreatic duct [2]. Endoscopic intervention is the treatment of choice. Worms visible at the papilla are removed endoscopically using a basket or forceps [3]. To conclude, biliary ascariasis should be considered in the differential diagnosis of IRAP, particularly in endemic regions.

Endoscopy_UCTN_Code_CCL_1AF_2AZ

\section{Competing interests}

None

The Authors

Malay Sharma, Piyush Somani,

Rajendra Prasad, Saurabh Jindal

Department of Gastroenterology, Jaswant Rai

Speciality Hospital, Saket, India
Corresponding author

Malay Sharma, MD, DM

Department of Gastroenterology, Jaswant Rai Speciality Hospital, Saket, Meerut, PIN-250 001, Uttar Pradesh, India Phone: +91-9837031148

Fax: +91-121-2657154

sharmamalay@hotmail.com

\section{References}

[1] Somani P, Sharma M, Pathak A et al. Endoscopic ultrasound imaging of pancreatic duct ascariasis. Endoscopy 2016; 48: E1 -E6

[2] Sharma M, Somani P, Patil A. Double trouble in acute pancreatitis. Gastroenterology 2016; 150: 833-834

[3] Krige J, Shaw J. Cholangitis and pancreatitis caused by biliary ascariasis. Clin Gastroenterol Hepatol 2009; 7: A30

\section{Bibliography}

DOI http://dx.doi.org/10.1055/s-0043-104523

Endoscopy 2016; 49: E143-E144

(c) Georg Thieme Verlag KG

Stuttgart $\cdot$ New York

ISSN 0013-726X 